\title{
Mucosal Integrity Testing Can Detect Differences in the Rectums of Patients with Inflammatory Bowel Disease Compared to Controls: A Pilot Study
}

\author{
Miles Basil ${ }^{1} \cdot$ David Schwartz $^{1} \cdot$ Robin Dalal $^{1} \cdot$ Sara Horst $^{1} \cdot$ Elizabeth Scoville $^{1}$ - Dawn Adams ${ }^{1} \cdot$ Dawn Beaulieu $^{1}$. \\ James C. Slaughter ${ }^{3} \cdot$ Tina Higginbotham $^{1} \cdot$ Michael Vaezi $^{1} \cdot$ Yash Choksi $^{1,2}$
}

Received: 21 December 2020 / Accepted: 1 February 2021 / Published online: 27 February 2021

(c) This is a U.S. government work and not under copyright protection in the U.S.; foreign copyright protection may apply 2021

\begin{abstract}
Background While the pathogenesis of inflammatory bowel disease (IBD) is incompletely understood, disruption of epithelial integrity is suspected to play a prominent role in disease initiation and progression. Currently, there is no convenient way to measure this in vivo.

Aims Our aim is to determine whether a mucosal integrity (MI) testing device that has been used to measure MI in the esophagus can also be used to measure barrier function in the colon during colonoscopy.

Methods Mucosal integrity testing was measured in patients with IBD $(n=17)$ and controls $(n=7)$ during colonoscopy. During the procedure, an MI catheter was passed down the working channel of the colonoscope and placed along the mucosal wall to measure MI in the rectum, left, transverse, and right colon. In patients with IBD, MI measurements and biopsies were taken in areas which appeared inflamed when present. We then determined if there was a significant difference in MI between patients with IBD and controls.

Results MI was significantly higher in the rectum of patients with IBD (CD and UC combined) versus control colons [767 $(618-991)$ vs. 531 (418-604) ohms, $P<0.01]$. There were no significant differences in MI among patients with IBD versus controls in the right, transverse, or left colon. Within the IBD group, there were no significant differences in MI between inflamed versus non-inflamed rectums. There was no correlation between quality of life scores or endoscopic severity with MI, though this study was likely underpowered to detect these differences.

Conclusion Rectal MI is significantly higher in patients with IBD versus controls. Future studies are needed to determine how this information can be used clinically.
\end{abstract}

Keywords Inflammatory bowel disease $\cdot$ Mucosal integrity testing $\cdot$ Intestinal barrier function · Endoscopy

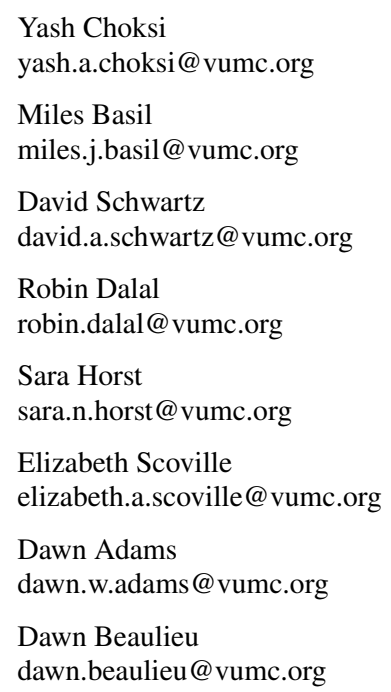

\author{
James C. Slaughter \\ james.c.slaughter@vumc.org \\ Tina Higginbotham \\ tina.higginbotham@vumc.org \\ Michael Vaezi \\ michael.vaezi@vumc.org
}

1 Division of Gastroenterology, Hepatology and Nutrition, Vanderbilt University Medical Center, 1076 Light Hall, 22nd Ave., South Nashville, TN 37232-5280, USA

2 Veterans Affairs, Tennessee Valley Health Care System, Nashville, TN, USA

3 Department of Biostatistics, Vanderbilt University School of Medicine, Vanderbilt School of Medicine, 2525 West End, Suite 1100, Nashville, TN 37203, USA 


\section{Introduction}

While the pathogenesis of inflammatory bowel disease (IBD) is incompletely understood, disruption of colonic epithelial integrity plays a prominent role in disease initiation and progression [1-4]. However, there is currently no convenient and efficient way to measure intestinal barrier function during endoscopy. Prior methods for testing intestinal permeability all have significant limitations. The lactulose/mannitol ratio (LAMA) which utilizes a large molecule (lactulose) that cannot pass through normal mucosa, measures small intestinal but not colonic permeability [5]. Confocal endomicroscopy makes use of images taken during colonoscopy to measure epithelial integrity [6-9]. Abnormalities captured by this technology have been shown to correlate with ongoing symptoms of diarrhea in patients with IBD, even after endoscopic healing [10]. Imaging modalities such as these, however, require injection of a permeability probe during the procedure and significant, time-consuming image analysis by an expert post procedurally. Mucosal integrity (MI) testing, which has been used in the esophagus to detect decreased epithelial integrity characteristic of gastroesophageal reflux disease (GERD) and eosinophilic esophagitis (EoE), provides an efficient, real-time measure of epithelial resistance during endoscopy [11-13]. Thus, we reasoned that MI may also be able to measure colonic mucosal integrity in IBD. As such, our aim was to determine whether mucosal integrity testing could detect changes in epithelial integrity in IBD.

\section{Methods}

The study was performed in accordance with the Declaration of Helsinki, Good Clinical Practice, and applicable regulatory requirements. Each patient signed a consent form before undergoing any study-related procedures. The Vanderbilt Institutional Review Board approved this clinical trial (IRB\# 160534), and it was registered at www.clinicaltrials. gov (NCT03165058). All authors had access to the study data and reviewed and approved the final manuscript.

\section{Study Design and Patient Population}

This is a prospective study in which patients were enrolled at the time of colonoscopy from August 2020 to October 2020. Inclusion criteria for patients with IBD included age over 18 and a prior histologic diagnosis of ulcerative colitis (UC) or Crohn's Disease (CD). Patients who had undergone prior small bowel surgeries or ileocecal resections were included. Exclusion criteria for patients with IBD included patients on experimental IBD therapy, pregnant patients, patients with indeterminate colitis, and patients who had undergone colonic resection. Indication for colonoscopy in the IBD group was either disease surveillance or worsening symptoms. Control subjects were patients who were undergoing average risk colon cancer screening without a known history of IBD. One control patient had a colonoscopy performed for gastrointestinal symptoms and was found to have a normal examination endoscopically (i.e., no evidence of inflammatory bowel disease).

\section{Equipment}

Mucosal integrity testing is currently being utilized at Vanderbilt University Medical Center for the assessment of esophageal and small bowel epithelial integrity [11-17]. A previously described MI catheter was used to measure electrical resistance of the esophageal epithelium by direct mucosal contact (InSight; Sandhill Scientific, Inc). Data were viewed and analyzed on BioView Analysis software (Sandhill Scientific, Inc) [14].

\section{Testing Procedure}

During colonoscopy, an MI catheter was inserted through the biopsy channel of a colonoscope. Once inserted, the endoscopist gently rested the probe against the targeted colonic mucosa for five seconds. Measurements were taken at four sites: right colon, transverse colon, left colon, and rectum. Within each of these segments, measurements were preferentially taken at the site of what appeared to be the most severe inflammation as determined endoscopically. Care was taken to take measurements at sites with minimal fluid and maximum contact with colonic mucosa. The majority of the procedures were completed by a single proceduralist, although a total of 3 endoscopists participated. The primary author (M.B.) was present in all procedures and was responsible for data collection and management of probe hardware and software throughout the study.

\section{Endoscopic Assessment of Disease Activity}

Previously validated scoring systems were utilized for measuring endoscopic disease activity. The Mayo score was used to evaluate disease activity in UC and the simplified endoscopic activity score for Crohn's disease (SES-CD) was used to endoscopically evaluate $\mathrm{CD}[18,19]$. The complete Mayo score typically utilizes stool frequency, rectal bleeding, physician global assessment, and endoscopic findings. In this case, we used only the endoscopic scoring system and applied a score of 0 to 3 to test subjects. Scores ranged from 0 (normal or inactive disease) to 3 (severe disease with spontaneous bleeding, ulceration). The SES-CD scoring system 
assesses the following variables in the ileum, right colon, transverse colon, left colon and rectum: presence and size of ulcers, extent of ulceration, extent of affected surface, and presence/type of stricture. Scores range from 0 (no disease) to 60 (severe disease).

\section{Pathology}

Biopsies were taken adjacent to the sites of mucosal integrity measurements. Histology was classified as normal, inactive/quiescent inflammation, mild inflammation, moderate inflammation, or severe inflammation based on expert GI pathologist review. We then assessed if MI values correlated with degree of inflammation on pathology. For this analysis, given the small sample size, we combined active disease (mild, moderate, severe) into one group and inactive disease into a second group (normal, inactive/quiescent).

\section{Measuring Symptoms}

Participants were asked on the day of endoscopy to rate their quality of life using the previously validated [20] short inflammatory bowel disease questionnaire (SIBDQ). The SIBDQ score is a tool used to measure physical, social, and emotional status in patients with IBD with scores ranging from 10 (low impact on quality of life) to 70 (high impact on quality of life). We assessed for correlation between patient's SIBDQ scores and MI values.

\section{Statistical Analysis}

Continuous variables were summarized using the median and interquartile range, and categorical variables were summarized using percentages. To test differences between groups, we used the nonparametric Kruskal-Wallis test for continuous variables and Pearson's Chi-squared test for categorical variables. We had $80 \%$ probability (power) to detect a 1.3 standard deviation difference in MI between UC/CD and Control, a 1.5 standard deviation difference between UC and CD patients. In supplementary analyses, simple linear regression was used to estimate the association between disease severity and quality of life (Mayo or SIBDQ score) and MI measurements. All analyses were conducted using a 2-sided significance level of 0.05 with the $\mathrm{R}$ statistics language [21].

\section{Results}

\section{Demographics}

Patient demographics are shown in Table 1 (Crohn's and UC patients separated) and Table 2 (patients with IBD as one group). MI testing was performed in 24 patients ( 9 with ulcerative colitis [UC], 8 with Crohn's Disease [CD] and 7 controls) during colonoscopy. The Crohn's disease patients were significantly younger than UC and controls. The IBD group had a higher rate of past GI surgical history. The average number of years since diagnosis for $\mathrm{UC}$ and $\mathrm{CD}$ was 7 and 11, respectively. Table 3 depicts the location and incidence of inflammation severity at a given segment of colon. There were no procedural complications in either the test groups or the control group.

\section{Measurements}

There was no significant difference in mucosal integrity in UC vs. Crohn's disease (Table 4). Thus, we combined the two groups for comparisons with the control group. MI was significantly higher in the rectum of patients with IBD versus control colons (Table 5, 767 [618-991] vs. 531 [418-604] ohms, $P<0.01)$. There was not a statistically significant difference in MI between IBD and controls in the right, transverse, or left colon (Tables 4, 5). There was not a significant difference in rectal MI between patients with active (mild, moderate, severe) versus inactive (normal, inactive/quiescent) inflammation by histology (Fig. 1). There was no significant correlation between rectal mucosal integrity and SIBDQ (Supplementary Fig. 1), Mayo Scores (Supplementary Fig. 2) in the UC patients or SES-CD scores in $\mathrm{CD}$ patients.

\section{Discussion}

Our results demonstrate that rectal MI is significantly higher in patients with IBD versus controls. This difference was not seen in the right, transverse, or left colon. Additionally, mucosal integrity values did not correlate with severity of inflammation, symptoms, or endoscopic inflammation. Since MI is lower in esophageal disease (e.g. GERD or EoE) where there is a loss of epithelial integrity, our initial hypothesis was that MI would be lower in patients with IBD for the same reason. However, this was not the case in our study as patients with IBD had significantly higher MI in the rectum compared with controls. There are multiple factors which could explain these results. It is likely that there are varying degrees of mucosal layer thickness and intraluminal fluid between subjects, depending on both colonic preparation quality and degree of inflammation, which could have varying effects on mucosal integrity testing. Differences in mucosal type (squamous vs. columnar) may also partially explain the differences in previously described MI values measured in the esophagus. This pattern is similar to prior findings which showed a significantly higher mucosal 
Table 1 Demographics for all patients by group (Controls, $\mathrm{UC}, \mathrm{CD})$ and medication use

\begin{tabular}{|c|c|c|c|c|}
\hline & $\begin{array}{l}\text { UC } \\
n=9\end{array}$ & $\begin{array}{l}\mathrm{CD} \\
n=8\end{array}$ & $\begin{array}{l}\text { Control } \\
N=7\end{array}$ & $P$ value \\
\hline Age (median) & $48(45,57)$ & $32(26,37)$ & $62(56,67)$ & $0.0011^{1}$ \\
\hline Gender & & & & $0.882^{2}$ \\
\hline Male $(n)$ & 4 & 4 & 4 & \\
\hline Female $(n)$ & 5 & 4 & 3 & \\
\hline Average \# years since diagnosis & 7 & 11 & & $0.613^{3}$ \\
\hline History of previous bowel surgery (\%) & 0 & 40 & 0 & $0.04^{2}$ \\
\hline \multicolumn{5}{|l|}{ Indication for endoscopy (\%) } \\
\hline CRC screening (\%) & & & 86 & $<0.001^{2}$ \\
\hline Disease surveillance (\%) & 56 & 38 & & \\
\hline Worsening symptoms (\%) & 44 & 62 & 14 & \\
\hline Number of procedural complications & 0 & 0 & 0 & \\
\hline $\begin{array}{l}\text { Average Symptoms of IBD questionnaire } \\
\text { (SIBDQ) score (range 10-70) }\end{array}$ & 55 & 48 & & $0.173^{3}$ \\
\hline Average SES-CD score & & $6.5(4.0,11.2)$ & & \\
\hline Mayo score of 0 & $11 \%(1 / 9)$ & & & \\
\hline Mayo score of 1 & $78 \%(7 / 9)$ & & & \\
\hline Mayo score of 2 & $11 \%(1 / 9)$ & & & \\
\hline Mayo score of 3 & $0 \%(0 / 9)$ & & & \\
\hline 5'ASA (\%) & 78 & 38 & & \\
\hline Topical steroids (\%) & 33 & 12 & & \\
\hline Azathiaprine (\%) & 0 & 12 & & \\
\hline Methotrexate (\%) & 11 & 0 & & \\
\hline Biologic therapy (\%) & 33 & 62 & & \\
\hline Systemic steroids (\%) & 11 & 12 & & \\
\hline
\end{tabular}

Continuous variables are represented as median (lower quartile, upper quartile). $\mathrm{n}$ is the number of nonmissing values. Tests used: ${ }^{1}$ Kruskal-Wallis test; ${ }^{2}$ Pearson test; ${ }^{3}$ Wilcoxon test

\begin{tabular}{llll}
\hline & $\begin{array}{l}\text { IBD (UC+Crohn's) } \\
n=17\end{array}$ & $\begin{array}{l}\text { Control } \\
n=7\end{array}$ & $P$ value \\
\hline $\begin{array}{l}\text { Age (median) } \\
\text { Gender }\end{array}$ & $40(27,48)$ & $62(56,67)$ & $0.007^{1}$ \\
Male $(n)$ & 8 & 4 & $0.65^{2}$ \\
Female $(n)$ & 9 & 3 & \\
Average \# years since diagnosis & 8 & $\mathrm{NA}$ & \\
History of previous bowel surgery $(\%)$ & 14 & 0 & $<0.001^{2}$ \\
Indication for colonoscopy $(\%)$ & & & \\
CRC screening $(\%)$ & 0 & 86 & \\
Disease surveillance $(\%)$ & 47 & 0 & 14 \\
Worsening symptoms $(\%)$ & 53 & & \\
\hline
\end{tabular}

Continuous variables are represented as median (lower quartile, upper quartile). $\mathrm{n}$ is the number of nonmissing values. Tests used: ${ }^{1}$ Kruskal-Wallis test; ${ }^{2}$ Pearson test
Table 2 Demographics for all patients when combining patients with UC and CD integrity in the duodenums of patients with active celiac disease versus control subjects [16]. It is possible that increased edema, mucosal thickness, or increased mucus production in the small intestine in celiac disease or colon in IBD can lead to higher MI values.
Further studies at the cellular and subcellular level are needed to further understand potential physiologic mechanisms for this observed $\mathrm{MI}$ difference in the rectum and to understand why this difference was not seen in other portions of the colon. Mucosal healing in IBD is a complex process 
Table 3 Histologic Inflammation severity based on colonic location

\begin{tabular}{lllll}
\hline & Right colon & Transverse & Left colon & Rectum \\
\hline$U C$ & & & & \\
Normal & $56 \%(5 / 9)$ & $33 \%(3 / 9)$ & $11 \%(1 / 9)$ & $11 \%(1 / 9)$ \\
Mild & $22 \%(2 / 9)$ & $11 \%(1 / 9)$ & $11 \%(1 / 9)$ & $22 \%(2 / 9)$ \\
Moderate & $0 \%(0 / 9)$ & $11 \%(1 / 9)$ & $22 \%(2 / 9)$ & $11 \%(1 / 9)$ \\
Severe & $0 \%(0 / 9)$ & $0 \%(0 / 9)$ & $11 \%(1 / 9)$ & $11 \%(1 / 9)$ \\
Inactive & $22 \%(2 / 9)$ & $44 \%(4 / 9)$ & $44 \%(4 / 9)$ & $44 \%(4 / 9)$ \\
$C D$ & & & & \\
Normal & $63 \%(5 / 8)$ & $50 \%(4 / 8)$ & $63 \%(5 / 8)$ & $63 \%(5 / 8)$ \\
Mild & $13 \%(1 / 8)$ & $13 \%(1 / 8)$ & $0 \%(0 / 8)$ & $0 \%(0 / 8)$ \\
Moderate & $13 \%(1 / 8)$ & $25 \%(2 / 8)$ & $13 \%(1 / 8)$ & $25 \%(2 / 8)$ \\
Severe & $13 \%(1 / 8)$ & $0 \%(0 / 8)$ & $13 \%(1 / 8)$ & $0 \%(0 / 8)$ \\
Inactive & $0 \%(0 / 8)$ & $0 \%(0 / 8)$ & $13 \%(1 / 8)$ & $13 \%(1 / 8)$ \\
\hline
\end{tabular}

with cellular, intracellular, and intercellular components at play simultaneously. Disruption in epithelial architecture, the extracellular matrix, and cytokine signaling is a function of degree and extent of inflammation, which is variable throughout the colon in patients with IBD [22, 23]. Thus, it is possible that a probe such as this is measuring a focal area of mucosal integrity rather than measuring barrier function of an entire segment of mucosa. A novel balloon MI catheter may decrease measurement variability by incorporating both radial and axial sensors mounted on a balloon to measure mucosal integrity along a longer segment of colonic mucosa, taking multiple measurements simultaneously [24]. Further research is needed using this new device.

Our study elucidated a significant difference in the rectums of patients with IBD versus control patients that was not significant in other colonic segments. Notably, there are differences in MI within the control group between the right side of the colon and the left side of the colon, suggesting that there are differences in the epithelium throughout the colon even in the absence of disease. One possible reason for this difference might be that the rectum is more susceptible to edema, resulting in variation in water and mucus content which might affect MI. However, it is unclear as to what the true etiology of these differences, and future studies are needed to understand these differences.
Table 5 Mucosal integrity (ohms) values in $\mathrm{UC}, \mathrm{CD}$ versus control colons (UC+CD combined)

\begin{tabular}{llll}
\hline & $\begin{array}{l}\text { IBD }(\mathrm{UC}+\mathrm{CD}) \\
N=17\end{array}$ & $\begin{array}{l}\text { Control } \\
N=7\end{array}$ & $P$ value \\
\hline MI right colon & $1139(864,1347)$ & $849(716,1250)$ & 0.29 \\
MI transverse colon & $891(705,1123)$ & $783(752,880)$ & 0.73 \\
MI left colon & $673(547,763)$ & $699(594,740)$ & 0.97 \\
MI rectum & $767(618,991)$ & $531(418,604)$ & 0.009 \\
\hline
\end{tabular}

Continuous variables are represented as median (lower quartile, upper quartile). $\mathrm{N}$ is the number of non-missing values. Tests used: Wilcoxon test

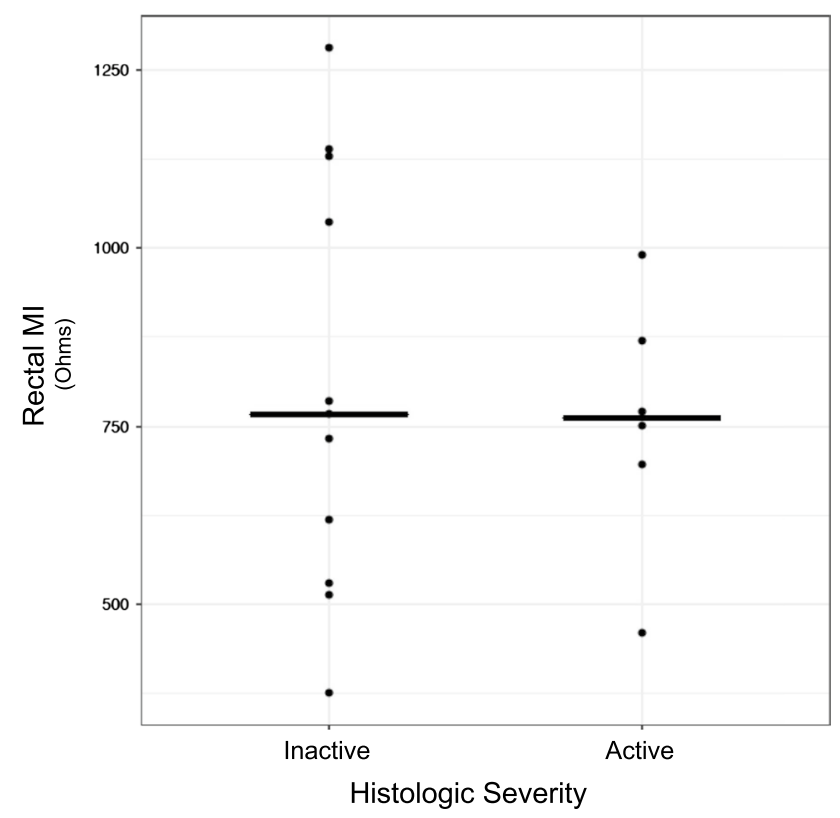

Fig. 1 Rectal MI is not significantly different in patients with IBD with active versus inactive disease $(P=0.81)$. Severity of histologic disease activity in the rectum did not correlate with rectal MI values. Active disease included mild, moderate, and severe disease, while inactive disease included inactive/quiescent disease and normal

Our study has multiple strengths. First, the mechanism for obtaining mucosal integrity measurements is procedurally simple and easily employed during routine
Table 4 Mucosal integrity (ohms) values in $\mathrm{UC}, \mathrm{CD}$ versus control colons

\begin{tabular}{lllll}
\hline & UC & CD & Control & $P$ value \\
& $N=9$ & $N=8$ & $N=7$ & \\
\hline MI right colon & $1014(805,1347)$ & $1206(1066,1294)$ & $849(716,1250)$ & 0.45 \\
MI transverse colon & $753(670,1019)$ & $974(844,1180)$ & $783(752,880)$ & 0.33 \\
MI left colon & $673(549,749)$ & $694(538,795)$ & $699(594,740)$ & 1 \\
MI rectum & $785(529,991)$ & $759(704,838)$ & $531(418,604)$ & 0.034 \\
\hline
\end{tabular}

Continuous variables are represented as median (lower quartile, upper quartile). $\mathrm{N}$ is the number of nonmissing values. Test used: Wilcoxon test 
colonoscopy for endoscopists of varying skill levels. This technology requires only a few added seconds per procedure and adds no risk to the patient. Developing an easily utilized technology such as this for a significantly prevalent condition such as IBD would prove extremely beneficial. Additionally, our study was able to assess multiple commonly employed markers of disease activity: symptomatology, endoscopic appearance, and histology.

Our study has multiple limitations to consider as well. The small sample size limits our ability to answer several questions, such as whether there is a difference between Crohn's and Ulcerative Colitis, whether or not mucosal integrity testing correlates with symptoms or endoscopic mucosal healing, or whether different drug therapies can alter MI values irrespective of disease activity. Unfortunately, the specific probe model used in this study was discontinued mid-study, limiting ability to obtain larger sample size. Determining whether there was a correlation between symptoms and mucosal integrity would be helpful clinically. For example, if larger studies revealed a potential correlation between IBD symptoms or quality of life and MI, developing a reliable and easily performed MI testing maneuver could provide valuable clinical data regarding the inflammatory status of an IBD patient. Furthermore, symptoms in IBD can be unrelated to level of inflammation and thus further assessing for inflammation with tools like fecal calprotectin and CRP may help further delineate level of inflammation, symptoms, and MI differences.

Furthermore, our results revealed a significant age difference between our control patients and patients with IBD. This raises the possibility that younger age may result in higher MI values in the rectum rather than presence of disease. The correlation between age and mucosal integrity with all groups combined did reveal a moderate and negative association between rectal mucosal integrity and age. However, when looking at each group (UC, Crohn's, controls) individually, there is no significant correlation between rectal MIT and age. Thus, while it is possible that higher rectal MIT in UC and Crohn's is at least partially due to age differences, our sample size is not big enough to make this conclusion. Notably, our group's prior studies in the esophagus have not shown any significant correlation in MIT values with age $[11,12$, 14-17]. Additionally, this correlation was not significant in other colonic segments. Future studies are required to understand the relationship between age and colonic MI.

In conclusion, while this device can detect a difference in impedance measurements in the rectum of patients with IBD as compared with controls, future studies are needed to answer whether this novel technology has clinical utility and whether it can be used to measure disease activity.
Supplementary Information The online version contains supplementary material available at https://doi.org/10.1007/s10620-021-06888-5.

Funding This work was supported by Department of Veterans Affairs Career Development Award IK2BX004648, Vanderbilt Digestive Disease Research Center Pilot Grant P30058404, a Vanderbilt SCRIPS Burroughs Welcome Fund Fellowship (to Y.A.C.)

\section{Compliance with Ethical Standards}

Conflict of interest Vanderbilt University and Diversatek Healthcare Inc. (Denver, CO, USA) jointly hold a patent on the mucosal integrity (MI) concept and device. This was disclosed to patients. Dr. Vaezi (MV) has had research funding from Diversatek Healthcare in the conduct of studies with mucosal integrity. Diversatek Healthcare had no influence on the study design, conduct, analysis or the final manuscript. There are no financial relationships between any of the other authors and Diversatek Healthcare Inc. All other authors have no conflicts of interest.

Open Access This article is licensed under a Creative Commons Attribution-NonCommercial 4.0 International License, which permits any non-commercial use, sharing, adaptation, distribution and reproduction in any medium or format, as long as you give appropriate credit to the original author(s) and the source, provide a link to the Creative Commons licence, and indicate if changes were made. The images or other third party material in this article are included in the article's Creative Commons licence, unless indicated otherwise in a credit line to the material. If material is not included in the article's Creative Commons licence and your intended use is not permitted by statutory regulation or exceeds the permitted use, you will need to obtain permission directly from the copyright holder. To view a copy of this licence, visit http://creativecommons.org/licenses/by-nc/4.0/.

\section{References}

1. Michielan A, D'Incà R. Intestinal Permeability in Inflammatory Bowel Disease: Pathogenesis, Clinical Evaluation, and Therapy of Leaky Gut. Mediators Inflamm. 2015;2015:628157. https://doi. org/10.1155/2015/628157.

2. Mankertz J, Schulzke J-D. Altered permeability in inflammatory bowel disease: pathophysiology and clinical implications. CurrOpinGastroenterol. 2007;23:379-383. https://doi.org/10.1097/ mog.0b013e32816aa392.

3. Antoni L, Nuding S, Wehkamp J, Stange EF. Intestinal barrier in inflammatory bowel disease. World J Gastroenterol. 2014;20:1165-1179. https://doi.org/10.3748/wjg.v20.i5.1165.

4. Jäger S, Stange EF, Wehkamp J. Inflammatory bowel disease: an impaired barrier disease. Langenbeck's Arch Surg. 2013;398:112. https://doi.org/10.1007/s00423-012-1030-9.

5. D'Incà R, Di Leo V, Corrao G, et al. Intestinal permeability test as a predictor of clinical course in Crohn's disease. Am J Gastroenterol. 1999;94:2956-2960.

6. Li CQ, Xie XJ, Yu T et al. Classification of inflammation activity in ulcerative colitis by confocal laser endomicroscopy. Am J Gastroenterol 2010;105:1391-1396

7. Liu J, Dlugosz A, Neumann H. Beyond white light endoscopy: the role of optical biopsy in inflammatory bowel disease. World $J$ Gastroenterol 2013;19:7544-7551 
8. Kiesslich R, Duckworth CA, Moussata D et al. Local barrier dysfunction identified by confocal laser endomicroscopy predicts relapse in inflammatory bowel disease. Gut 2012;61:1146-1153

9. Neumann H, Vieth M, Atreya R, Neurath MF, Mudter J. Prospective evaluation of the learning curve of confocal laser endomicroscopy in patients with IBD. Histol Histopathol 2011;26:867-872

10. Chang J, Leong RW, Wasinger VC, Ip M, Yang M, Phan TG. Impaired intestinal permeability contributes to ongoing bowel symptoms in patients with inflammatory bowel disease and mucosal healing. Gastroenterology 2017;153:723-731.e1. https ://doi.org/10.1053/j.gastro.2017.05.056.

11. Ates F, Yuksel ES, Higginbotham $T$ et al. Mucosal impedance discriminates GERD from non-GERD conditions. Gastroenterology 2015;148:334-343

12. Choksi YA, Chaparro J, Blanco M, Sharda R, Sarker S, Ferguson et al. The sub-UES Esophageal Region is distinct in active eosinophilic esophagitis and may distinguish EoE from Lichen Planus. Clin Gastroenterol Hepatol. 2019. https://doi. org/10.1016/j.cgh.2019.09.032.

13. Barrett C, Choksi Y, Vaezi MF. Mucosal impedance: a new approach to diagnosing gastroesophageal reflux disease and eosinophilic esophagitis. CurrGastroenterol Rep. 2018;20:33

14. Saritas Yuksel E, Higginbotham T, Slaughter JC et al. Use of direct, endoscopic-guided measurements of mucosal impedance in diagnosis of gastroesophageal reflux disease. Clin Gastroenterol Hepatol 2012;10:1110-1116

15. Katzka DA, Ravi K, Geno DM et al. Endoscopic mucosal impedance measurements correlate with eosinophilia and dilation of intercellular spaces in patients with eosinophilic esophagitis. Clin Gastroenterol Hepatol 2015; 13:1242-1248

16 Adams DW, Patel D, Evers L, Slaughter JC, Higginbotham T, Vaezi M. Measurements of duodenal mucosal integrity are altered in celiac disease. ClinGastroenterolHepatol. 2019. https://doi. org/10.1016/j.cgh.2019.07.032.

17. Saritas Yuksel E, Higginbotham T, Slaughter JC, et al. Use of direct, endoscopic-guided measurements of mucosal impedance in diagnosis of gastroesophageal reflux disease. Clin Gastroenterol Hepatol. 2012;10:1110-1116. https://doi.org/10.1016/j. cgh.2012.05.018.

18 Schroeder KW, Tremaine WJ, Ilstrup DM. Coated oral 5-aminosalicylic acid therapy for mildly to moderately active ulcerative colitis: a randomized study. $N$ Engl J Med. 1987;317:1625-1629. https://doi.org/10.1056/NEJM198712243172603.

19. Daperno M, D'Haens G, Van Assche G et al. Development and validation of a new, simplified endoscopic activity score for Crohn's disease: the SES-CD. Gastrointest Endosc. 2004;60:505512. https://doi.org/10.1016/s0016-5107(04)01878-4.

20. Jowett SL, Seal CJ, Barton JR, Welfare MR. The short inflammatory bowel disease questionnaire is reliable and responsive to clinically important change in ulcerative colitis. Am J Gastroenterol. 2001;96:2921-2928.

21. R Core Team. $R$ : a language and environment for statistical computing. Vienna: R Foundation for Statistical Computing; 2020.

22. Luissint AC, Parkos CA, Nusrat A. Inflammation and the Intestinal Barrier: Leukocyte-Epithelial Cell Interactions, Cell Junction Remodeling, and Mucosal Repair. Gastroenterology. 2016;151:616-32. https://doi.org/10.1053/j.gastro.2016.07.008.

23 Ho G, Cartwright JA, Thompson EJ, Bain CC, Rossi AG. Resolution of inflammation and gut repair in IBD: translational steps towards complete mucosal healing. Inflam Bowel Dis. 2020. https ://doi.org/10.1093/ibd/izaa045.

24. Patel DA, Higginbotham T, Slaughter JC, Aslam M, Yuksel E, Katzka D et al. Development and validation of a mucosal impedance contour analysis system to distinguish esophageal disorders. Gastroenterology. 2019 May;156:1617-1626.e1. https://doi. org/10.1053/j.gastro.2019.01.253.

Publisher's Note Springer Nature remains neutral with regard to jurisdictional claims in published maps and institutional affiliations. 